\title{
On finite dimensional realizations for the term structure of futures prices
}

\author{
Tomas Björk, \\ Department of Finance, \\ Stockholm School of Economics, \\ Box 6501, \\ SE-113 83 Stockholm, \\ SWEDEN \\ e-mail: tomas.bjork@hhs.se
}

\author{
Magnus Blix, \\ Department of Finance, \\ Stockholm School of Economics, \\ Box 6501, \\ SE-113 83 Stockholm, \\ SWEDEN \\ e-mail: magnus.blix@hhs.se
}

\author{
Camilla Landén, \\ Department of Mathematics, \\ Royal Institute of Technology, \\ SE-100 44 Stockholm, \\ SWEDEN \\ e-mail: camilla@math.kth.se
}

September 20, 2005

To appear in

International Journal of Theoretical and Applied Finance

\begin{abstract}
We consider HJM type models for the term structure of futures prices, where the volatility is allowed to be an arbitrary smooth functional of the present futures privce curve. Using a Lie algebraic approach we investigate when the infinite dimensional futures price process can be realized by a finite dimensional Markovian state space model, and we give general necessary and sufficient conditions, in terms of the volatility structure, for the existence of a finite dimensional realization. We study a number of concrefe applications including a recently developed model for gas futures. In particular we provide necessary and sufficient conditions for when the induced spot price is a Markov process. In particular we can prove that the only HJM type futures price models with spot price dependent volatility structures which generically possess a spot price realization are the affine ones. These models are thus the only generic spot price models from a futures price term structure point of view.
\end{abstract}


Keywords HJM models, factor models, state space models, Markovian realizations

JEL Classification numbers E43

\section{Introduction}

When studying the term structure of futures prices, there has traditionally been two main approaches in the literature; the state space approach and HeathJarrow-Morton type approach. They can roughly be described as follows.

1. In the state space approach we start by modeling the dynamics of a finite dimensional Markovian state vector process $Z$. This is typically done in the form of a system of stochastic differential equations (hencforth SDEs) under a risk neutral martingale measure $Q$. The underlying asset price $S$ is then specified as $S_{t}=h\left(Z_{t}\right)$ for some deterministic function $h$, and very often $S$ is one of the components of $Z$ in which case $h$ is just a coordinate function. The futures price at $t$ for delivery at $T$ is then given by standard theory as

$$
F(t, T)=E^{Q}\left[S_{T} \mid \mathcal{F}_{t}\right] .
$$

From the Markovian structure it follows that we can write $F(t, T)=$ $H\left(t, Z_{t}, T\right)$ for some deterministic function $H$, and $H$ can finally be determined as the solution of a parabolic partial differential equation. Examples of this approach can be found in [1], [5], [16], and [24].

2. In the HJM type approach we do not have an exogenously given finite dimensional state vector process $Z$. Instead we view the entire term structure of futures prices (or equivalently the entire futures price cruve) as the primitive object and model all futures prices simulataneously. Observing that, because of (1), all futures prices are $Q$ martingales and restricting ourselves to nonnegative underlying claims, we can model futures prices as

$$
d F(t, T)=F(t, T) \sigma_{F}(t, T) d W_{t},
$$

where, for each fixed maturity $T$, the volatility $\sigma_{F}(t, T)$ is some exogenously given vector valued adapted process, $W$ is $Q$-Wiener and we use the observed term structure of today as the initial condition. This approach was introduced in [11] (referring to the unpublished paper [23]) and has also been used (with some variations) in [5] and in [21].

These approaches have obvious counterparts in interest rate theory, the first corresponding to multifactor models (including short rate models), whereas the second corresponds to the HJM framework for modelling forward rates.

As is the case in interest rate theory, a multifactor state space model will in a trivial way generate a HJM type model. In the other direction, however, 
there is in general no implication. The reason for this is that a HJM model of the form (2) is an infinite dimensional system of SDEs (one equation for each $T$ ), and it is obviously only for very particular choices of the volatility structure $\sigma_{F}(t, T)$ that such an infinite system can be represented by a state space model.

The purpose of the present paper is precisely to investigate under what conditions on the volatility structure $\sigma_{F}(t, T)$ the inherently infinite dimensional model (2) can be represented in terms of a finite dimensional state space model. In such a case we say that the model (2) admits a Finite Dimensional Realization (henceforth FDR).

The corresponding FDR problem for interest rate models was more or less completely solved by geometric arguments in a series of papers [4], [6], [7], [14], and the main technical tool is the Lie algebra theory developed in [6]. For an overview of the theory see [3]. In the present paper we adapt the geometric theory developed in the papers above to solve the FDR problem for futures prices.

The structure and results of the paper is as follows.

- In Section 2 we present the probabilistic setup and formulate the precise problem under study.

- In Section 3 we give a very general necessary and sufficient condition for the existence of an FDR. By specializing to the cases of deterministic volatility structures and "deterministic direction" volatility structures we obtain more concrete results.

- Section 4 is devoted to a brief discussion on so called invariant manifolds, which provide a detailed description of the possible shapes of the futures price curve, which can be produced by a given model.

- In Section 5 we provide an algorithm which, given the existence of an FDR, will actually construct a concrete state space realization.

- Time varying models (which thus allow for seasonality effects) are treated in Section 6. As an illustration of the theory we study, in some detail, a concrete model for gas futures.

- We finish the main part of the paper in Section 7 by clarifying exactly under what conditions a given HJM-type model admits a realization where the spot price acts as the single underlying factor.

- In Appendix A we have, for the convenience of the reader, collected the basic definitions and results from differential geometry that are needed for the present paper.

\section{Basics}

We consider a financial market living on a given filtered probability space $\left\{\Omega, \mathcal{F}, Q,\left\{\mathcal{F}_{t}\right\}_{t \geq 0}\right\}$ carrying an $m$-dimensional Wiener process $W$. The main 
assets to be considered are futures contracts written on a given underlying asset. Let $F(t, T)$ denote the futures price at time $t$ of a futures contract with delivery date $T$. A simple arbitrage argument then yields the following relationship between the futures prices and the induced spot price $S$

$$
S(t)=F(t, t) .
$$

We assume that the market is arbitrage free in the sense that the probability measure $Q$ is a risk-neutral martingale measure. From standard theory (see e.g. [2]) we have the following well-known result

$$
F(t, T)=E^{Q}\left[S(T) \mid \mathcal{F}_{t}\right] .
$$

From equation (4) it is clear that for every fixed $T$ the futures price process is a $Q$-martingale. Thus, considering only nonnegative claims, we may assume that the futures prices to have dynamics of the following form under $Q$

$$
d F(t, T)=F(t, T) \sigma_{F}(t, T) d W_{t},
$$

where for each $T$, the volatily $\sigma_{F}(t, T)$ is an exogenously given adapted $m$ dimensional row vector process.

The main problem to be considered in this paper is to characterize the volatilities $\sigma_{F}$ for which the solution of the infinite dimensional SDE (5), i.e. the SDE for the futures prices, possesses a finite dimensional realization (FDR).

However, before giving the precise definition of an FDR of the futures prices model (5), it is necessary to rewrite (5) on a form which is more convenient for our purposes, and we start by reparameterizing it. We thus choose to parameterize the futures prices in terms of $t$ and $x$, where $x$ denotes time to maturity in contrast to $T$, which denotes time of maturity (compare with the Musiela parameterization of the forward rates, [9] and [22]). Therefore, let $F_{0}(t, x)$ be defined by

$$
F_{0}(t, x)=F(t, t+x) .
$$

It is then relatively easy to see that the process $F_{0}$ will have the following induced dynamics

$$
d F_{0}(t, x)=\frac{\partial}{\partial x} F_{0}(t, x) d t+F_{0}(t, x) \sigma_{0}(t, x) d W_{t},
$$

where

$$
\sigma_{0}(t, x)=\sigma_{F}(t, t+x) .
$$

It turns out that the analysis is considerably simplified if, instead of working with the process $F_{0}$ defined in (6), we work with the process $q$ where $q$ is defined by

$$
q(t, x)=\ln F_{0}(t, x) .
$$

Using the Itô formula on (5) or (7), we obtain the SDE for $q$ as

$$
d q(t, x)=\left\{\frac{\partial}{\partial x} q(t, x)-\frac{1}{2}\left\|\sigma_{0}(t, x)\right\|^{2}\right\} d t+\sigma_{0}(t, x) d W_{t} .
$$

There are two ways in which we can view the system 10 : 
- We can view (10) as a coupled system of infinitely many scalar SDEs (one for each fixed $x$ ).

- Alternatively we can view (10) as a single SDE, descirbing the dynamics of an infinite dimensional object. The infinite dimensional object is of course the futures price curve $x \longmapsto q_{t}(x)$.

The second interpretation turns out to much more fruitful for our purposes, so from now on we will denote the entire futures price curve at time $t$, i.e. the curve $x \longmapsto q_{t}(x)$, by the synbol $q_{t}$, and we can thus view $q_{t}$ as a point or vector in an infinite dimensional function space $\mathcal{H}$. It then remains to specify a suitable function space $\mathcal{H}$, and it turns out that it can in fact be chosen as a Hilbert space. See [6] or [14] for details.

In order to have a Markovian structure (albeit infinite dimensional) we make the following assumption.

Assumption 2.1 We assume that the futures price volatilty process is of the form

$$
\sigma_{0}(t, x)=\sigma\left(q_{t}, x\right),
$$

where $\sigma: \mathcal{H} \times R_{+} \rightarrow R^{m}$ is an exogensously given mapping.

Under this assumption, each component $\sigma_{i}$ (for $i=1, \ldots, m$ ) is a mapping $\sigma_{i}: \mathcal{H} \times R_{+} \rightarrow R$, i.e. a point $q \in \mathcal{H}$ and a nonegative real number $x \in R_{+}$ will be mapped into the real number $\sigma_{i}(q, x)$. We may however also, and more profitably, view $\sigma_{i}$ as a mapping between function spaces. More precisely: a point $q \in \mathcal{H}$ is mapped to the function $x \longmapsto \sigma_{i}(q, x)$. We will in fact assume that this function is a member of $\mathcal{H}$.

Assumption 2.2 We assume that the futures price volatilty mapping is of the form $\sigma: \mathcal{H} \rightarrow \mathcal{H}^{m}$, where each component $\sigma_{i}$ is a smooth vector field on $\mathcal{H}$.

We can now write the futures price equation (10) more compactly as

$$
d q_{t}=\left\{\mathbf{A} q_{t}-\frac{1}{2} D\left(q_{t}\right)\right\} d t+\sigma\left(q_{t}\right) d W_{t}
$$

where the vector fields $\mathbf{A}$ and $d$ are given by

$$
\begin{aligned}
\mathbf{A} & =\frac{\partial}{\partial x}, \\
D(q) & =\|\sigma(q)\|_{R^{m}}^{2}=\sum_{i=1}^{m} \sigma_{i}^{2}(q) .
\end{aligned}
$$

Finally, because of the geometrical ideas behind the results we will use, we need the Stratonovich form of the SDE (12). This is given by

$$
d q_{t}=\left\{\mathbf{A} q_{t}-\frac{1}{2} D\left(q_{t}\right)-\frac{1}{2} \sigma_{q}^{\prime}(q) \sigma(q)\right\} d t+\sigma\left(q_{t}\right) \circ d W_{t}
$$


where $\sigma_{q}^{\prime}(q) \sigma(q)$ denotes the Frechet derivative $\sigma_{q}^{\prime}(q)$ operating on $\sigma(q)$.

We can now specify exactly what we mean by a finite dimensional realization of (the logarithm of) the futures prices generated by a volatility $\sigma$. To this end fix a volatility $\sigma: \mathcal{H} \times R_{+} \rightarrow R^{m}$. We then have the following definition.

Definition 2.1 We say that the SDE (15) has a generic (local) d-dimensional realization at a given point $q^{0} \in \mathcal{H}$ if, for each initial point $q_{0}$ near $q^{0}$, there exists a point $z_{0} \in R^{d}$, smooth vector fields $a, b_{1}, \ldots, b_{m}$ on some open subset $\mathcal{Z}$ of $R^{d}$ and a smooth (submanifold) map $G: \mathcal{Z} \rightarrow \mathcal{H}$, such that $q$ has the local representation

$$
q_{t}=G\left(Z_{t}\right), \quad \text { a.s. }
$$

where $Z$ is the strong solution of the d-dimensional Stratonovich SDE

$$
\left\{\begin{aligned}
d Z_{t} & =a\left(Z_{t}\right) d t+b\left(Z_{t}\right) \circ d W_{t} \\
Z_{0} & =z_{0}
\end{aligned}\right.
$$

adn where the driving Wiener process $W$ in (17) is the same as in (15). The term "local" above means that the representation is assumed to hold for all $t$ with $0 \leq t \leq \tau\left(q^{0}\right)$, a.s. where, for each $q^{0} \in \mathcal{H}, \tau\left(q^{0}\right)$ is a strictly positive stopping time.

In slightly more pedestrian terms we can view the "output map" $G$ above as a mapping $G: \mathcal{H} \times R_{+} \rightarrow R$, so (16) can also be written as

$$
q_{t}(x)=G\left(Z_{t}, x\right) .
$$

Our main problems are now as follows.

- What are the necessary and sufficient conditions on the volatility structure $\sigma$ for the existence of a generic finite dimensional realizations?

- Suppose you know, from abstract theoy, that an FDR exists, how do you construct a concrete realization?

These problems will be solved in the next sections.

Remark 2.1 We have, for convenience, chosen to model the futures prices in terms of the log price $q$ and the corresppnding volatility $\sigma(q, x)$. We could of course also have chosen to model $F_{0}(t, x)$ and a corresponding volatility $\sigma_{0}\left(F_{0}, x\right)$. The volatilities correspond as follows

$$
\sigma_{0}\left(F_{0}, x\right)=\sigma\left(e^{q}, x\right)
$$

It is important to note that it may well happen that the SDE generated by $\{\mu, \sigma\}$ has a finite dimensional realization for a particular choice of initial point $q^{0}$, whereas no finite dimensional realization exists for points close to $q^{0}$. We say that such a system has a non-generic or "accidental" finite dimensional realization at $q^{0}$. If, on the other hand, the system has a finite dimensional 
realization for all points in a neighborhood of $q^{0}$ we say that the system has a generic finite dimensional realization at $r^{o}$. The existence of a non-generic realization is of course of very limited value, since the situation is structurally unstable. We note that our Lie algebraic result above guarantees the existence of a generic finite dimensional realization. In the sequel we will thus suppress the adjective "generic".

\section{Remark 2.2}

\section{Conditions for the existence of a finite dimen- sional realization}

In this section we give necessary and sufficient conditions for the term structure of futures prices to possess a finite dimensional realization. As it turns out, our present problem falls prefectly within the abstract framework developed in [6]. We need a simple nondegeneracy condition.

Assumption 3.1 Define the futures price drift vector field $\mu$ by

$$
\mu(q)=\mathbf{A} q_{t}-\frac{1}{2} D\left(q_{t}\right)-\frac{1}{2} \sigma_{q}^{\prime}(q) \sigma(q)
$$

with $\mathbf{A}$ and $D$ as above. We assume that the dimension of the Lie algebra $\left\{\mu, \sigma_{1}, \ldots, \sigma_{m}\right\}_{L A}$ is constant near the initial curve $q^{0}$.

We now have the following fundamental result.

Theorem 3.1 Take as given the volatility mapping $\sigma=\left(\sigma_{1}, \ldots, \sigma_{m}\right)$ as well as an initial curve $q^{0} \in \mathcal{H}$. Then the futures price model generated by $\sigma$ generically admits a finite dimensional realization at $q^{0}$, if and only if

$$
\operatorname{dim}\left\{\mu, \sigma_{1}, \ldots, \sigma_{m}\right\}_{L A}<\infty
$$

in a neighbourhood of $q^{0}$, where $\mu$ is given by equation (19).

Proof. Follows directly from Theorem 3.2. in [6].

This result solves completely, but in very abstract terms, the existence problem for finite dimensional erealisations. It is, however, not at all clear what the Lie algebra conditions means in concrete terms for the structure of $\sigma$. To obtain more concrete results we will therefore, in the next two sections, apply the abstract theorem to the special cases when the volatility is deterministic and when it has a constant direction. The arguments and results in these sections are very similar to those in [6]. 


\subsection{Deterministic volatility}

We start by considering the simplest case possible, i.e. when the volatility $\sigma$ is deterministic and we can write

$$
\sigma(q, x)=\sigma(x) .
$$

In geometric terms this means that each vector field $\sigma_{i}$ is a constant vector field.

As mentioned above, the realization problems that we study for futures price models have earlier been studied for forward interest rate models in [6]. In the present paper, the basic equation under study is the forward price equation (15) study, whereas the focus in [6] is on the HJM forward rate equation (with Musiela parameterization and on Stratonovich form). The main difference bewtween the two settings lies in the drift $\mu$. In the HJM forward rate framework the drift is given by

$$
\mu(r)=\frac{\partial}{\partial x} r+\sigma(r) \mathbf{H} \sigma(r)^{*}-\frac{1}{2} \sigma_{r}^{\prime}(r)[\sigma(r)] .
$$

Here $r$ denotes a generic forward rate curve $x \longmapsto r(x), \sigma(r, x)$ denotes the forward rate volatility, $*$ denotes transpose, and $\mathbf{H} \sigma$ is defined by

$$
\mathbf{H} \sigma(r, x)=\int_{0}^{x} \sigma(r, s) d s .
$$

Thus the main difference between the forward rate equation and the forward price equation is that the drift term $\sigma(r) \mathbf{H} \sigma(r)^{*}$, which is reklated to the HJM drift condition for forward rates, is replaced by the term $D(q)$ in the futures price equation. As a consequence of this, the arguments in the present paper are often more or less parallell to the corresponding arguments in [6]. In order to make the present paper self contained, we will however often give full arguments rather than referring the reader to parallell arguments in [6].

We may now state the main result for deterministic futures price volatilities.

Proposition 3.1 Assume that the volatility structure is of the form (20). Then the model possesses a finite dimensional realization if and only if the function space

$$
\operatorname{span}\left\{\frac{\partial^{k} \sigma_{i}}{\partial x^{k}} ; i=1, \ldots, m ; k=0,1, \ldots\right\}
$$

is finite dimensional.

Proof. For simplicity of notation we start by considering only the case with a scalar Wiener process so, referring to Theorem 3.1, we compute the Lie algebra $\mathcal{L}(\mu, \sigma)$. Since the vector field $\sigma$ is constant we have $\sigma^{\prime}=0$. Thus there is now Stratonovich correction term so from from (15) we have

$$
\mu(r)=\mathbf{F} q-\frac{1}{2} D,
$$


where as before $\mathbf{A}=\frac{\partial}{\partial x}$ and the vector field $D$ defined by $D(q, x)=\sigma^{2}(x)$ is a constant vector field. The Frechet derivatives are trivial in this case. Since $\mathbf{F}$ is linear and $D$ is constant, we obtain

$$
\begin{aligned}
& \mu^{\prime}=\mathbf{F}, \\
& \sigma^{\prime}=0 .
\end{aligned}
$$

Thus the Lie bracket $[\mu, \sigma]$ is given by

$$
[\mu, \sigma]=\mathbf{F} \sigma,
$$

and in the same way we have

$$
[\mu,[\mu, \sigma]]=\mathbf{F}^{2} \sigma .
$$

Continuing in the same way it is easily seen that the relevant Lie algebra $\mathcal{L}$ is given by

$$
\mathcal{L}=\{\mu, \sigma\}_{L A}=\operatorname{span}\left\{\mu, \sigma, \mathbf{F} \sigma, \mathbf{F}^{2} \sigma, \ldots\right\} .
$$

It is thus clear that $\mathcal{L}$ is finite dimensional (at each point $q$ ) if and only if the function space

$$
\operatorname{span}\left\{\mathbf{F}^{n} \sigma ; n=0,1,2, \ldots\right\}
$$

is finite dimensional. Recalling that $\mathbf{F}=\partial / \partial x$ finishes the proof, and the argument easily carries over to the case of several driving Wiener processes.

This result is stated in somewhat abstract terms, but we can easily obtain a much more concrete formulation. To do this we need the concept of a quasi exponential function.

Definition 3.1 A scalar real valued function of a real variable $x$ is called quasi exponential if it has the form

$$
f(x)=\sum_{i} e^{\lambda_{i} x}+\sum_{j} e^{\alpha_{j} x}\left[p_{j}(x) \cos \left(\omega_{j} x\right)+q_{j}(x) \sin \left(\omega_{j} x\right)\right],
$$

where $\lambda_{i}, \alpha_{j}, \omega_{j}$ are real numbers, whereas $p_{j}$ and $q_{j}$ are real polynomials.

We recall the following well known facts about quasi exponential functions.

Lemma 3.1 The following hold for the quasi-exponential functions.

- A function is $Q E$ if and only if it is a component of the solution of a vector valued linear ODE (ordinary differential equation) with constant coefficients.

- A function is $Q E$ if and only if it can be written as $f(x)=c e^{A x} b$.

- If $f$ is $Q E$, then $f^{\prime}$ is $Q E$.

- If $f$ is $Q E$, then its primitive function is $Q E$. 
- If $f$ and $g$ are $Q E$, then $f g$ is $Q E$. In particular, if $f$ is $Q E$, then so is $f^{2}$.

We now have the following very explicit formulation of Proposition 3.1.

Corollary 3.1 In the case of deterministic volatilities, the futures price eaqution possesses an FDR if and only if each component $\sigma_{i}(x)$ for $i=1, \ldots, m$ is a quasi exponential function.

Proof. From Proposition3.1 we know that the existence of an FDR is equivalent to the condition that

$$
\operatorname{span}\left\{\frac{\partial^{k} \sigma_{i}}{\partial x^{k}} ; i=1, \ldots, m ; k=0,1, \ldots\right\}
$$

is finite dimensional. This condition, on the other hand, is equivalent to to existence of linear relations between the various components of $\sigma$ and their derivatives. This, in turn, is equivalent to saying that each $\sigma_{i}$ is a component of the solution of a vector valued linear ODE with constant coefficients, and thus $\sigma_{i}$ is quasi exponential.

Remark 3.1 Since the volatility $\sigma(q, x)$ does not depend upon $q$, we have from remark 2.1 that $\sigma(x)=\sigma_{0}(x)$, and thus the conditons on $\sigma$ in Proposition 3.1 apply also to $\sigma_{0}$.

\subsection{Constant direction volatility}

The next simplest volatility structure you can consider is a volatility, where each component is of the form of the form

$$
\sigma_{i}(q, x)=\varphi_{i}(q) \lambda_{i}(x) .
$$

Here $\lambda_{i}$ is a constant vector field (it does not depned on $q$ ) whereas $\varphi_{i}$ is a smooth scalar field, i.e. a mapping $\varphi: \mathcal{H} \rightarrow R$. As a vector field we thus see that $\sigma_{i}$ has "constant direction" (namely the direction of $\lambda_{i}$ ) but that the length of $\sigma_{i}$ is modulated by the scalar field $\varphi_{i}$.

For the case when there is only a scalar driving Wiener process we obtain the following proposition, where we use the notation

$$
\Phi(q)=\varphi^{2}(q),
$$

and where $\Phi^{\prime \prime}(q)[\lambda ; \lambda]$ denotes the second order Frechet derivative of $\Phi$ evlauated at the point $q$, operating on the pair $(\lambda, \lambda) \in \mathcal{H}$. Notice the difference between the pair $[\lambda ; \lambda]$ with a semi colon), and the Lie bracket $[\lambda, \lambda]$ with a comma.

Proposition 3.2 Assume that the Wiener process is scalar, that $\varphi(q) \neq 0$ for all $q \in \mathcal{H}$, and that $\Phi^{\prime \prime}(q)[\lambda ; \lambda] \neq 0$ for all $q \in \mathcal{H}$. Then the futures price model has a finite dimensional realization if and only if $\lambda$ is a quasi-exponential function, whereas $\varphi$ is allowed to be any smooth field. 
Proof. In this case the drift vector field $\mu$ is given by

$$
\mu(q)=\mathbf{F} r-\frac{1}{2} \varphi^{2}(q) L-\frac{1}{2} \varphi^{\prime}(q)[\lambda] \varphi(q) \lambda,
$$

where $\varphi^{\prime}(q)[\lambda]$ denotes the Frechet derivative of $\varphi$ evaluated at $q$ and acting on the vector $\lambda$. The constant vector field $L \in \mathcal{H}$ is given by

$$
L(x)=\lambda^{2}(x) .
$$

We now want to prove that the Lie algebra generated by

$$
\begin{aligned}
\mu(q) & =\mathbf{F} q-\frac{1}{2} \varphi^{2}(q) L-\frac{1}{2} \varphi^{\prime}(q)[\lambda] \varphi(q) \lambda, \\
\sigma(q) & =\varphi(q) \lambda,
\end{aligned}
$$

is finite dimensional. Under the assumption that $\varphi(q) \neq 0$ for all $q$, we can use Lemma A.1, to see that the Lie algebra is in fact generated by the simpler system of vector fields

$$
\begin{aligned}
f_{0}(q) & =\mathbf{F} q-\frac{1}{2} \Phi(q) L, \\
f_{1}(q) & =\lambda
\end{aligned}
$$

where we have used the notation

$$
\Phi(q)=\varphi^{2}(q) .
$$

Since the field $f_{1}$ is constant, it has zero Frechet derivative, so the first Lie bracket is easily computed as

$$
\left[f_{0}, f_{1}\right](q)=\mathbf{F} \lambda+\Phi^{\prime}(q)[\lambda] L .
$$

The bracket $\left[\left[f_{0}, f_{1}\right], f_{1}\right]$ is easily obtained as

$$
\left[\left[f_{0}, f_{1}\right], f_{1}\right](q)=\Phi^{\prime \prime}(q)[\lambda ; \lambda] L .
$$

We may again use Lemma A.1 to see that the Lie algebra is generated by the following vector fields

$$
\begin{aligned}
& f_{0}(q)=\mathbf{F} q, \\
& f_{1}(q)=\lambda, \\
& f_{3}(q)=\mathbf{F} \lambda, \\
& f_{4}(q)=L .
\end{aligned}
$$

Of these vector fields, all but $f_{0}$ are constant, so all brackets are easy. After elementary calculations we see that in fact

$$
\{\mu, \sigma\}_{L A}=\operatorname{span}\left\{\mathbf{F}, \mathbf{F}^{n} \lambda, \mathbf{F}^{n} D ; n=0,1, \ldots\right\} .
$$


From this expression it follows immediately that a necessary condition for the Lie algebra to be finite dimensional is that the vector space spanned by $\left\{\mathbf{F}^{n} \lambda ; n \geq 0\right\}$ is finite dimensional. This occurs if and only if $\lambda$ is quasi-exponential If, on the other hand, $\lambda$ is quasi-exponential, then we know from Lemma 3.1, that also $L=\lambda^{2}$ is quasi-exponential. Thus the space $\left\{\mathbf{F}^{n} L ; n=0,1, \ldots\right\}$ is also finite dimensional, and we are finished.

We may also restate this result in terms of $F_{0}(t, x)$ and a volatility structure of the form $\sigma_{0}\left(F_{0}, x\right)=\varphi_{0}\left(F_{0}\right) \lambda(x)$ where $\lambda$ is the same constant field as in (22), and

$$
\varphi_{0}\left(F_{0}\right)=\varphi\left(\ln F_{0}\right) .
$$

Proposition 3.2 can now be rewritten in these new terms. If we let

$$
\Phi_{0}\left(F_{0}\right)=\varphi_{0}^{2}\left(F_{0}\right),
$$

we have the following proposition.

Proposition 3.3 Assume that $\varphi_{0}\left(F_{0}\right) \neq 0$ for all $F_{0} \in \mathcal{H}$, and that the condition $\Phi_{0}{ }^{\prime \prime}\left(F_{0}\right)[\lambda ; \lambda] \neq 0$ is satisfied for all $F_{0} \in \mathcal{H}$. Then the futures price model has a finite dimensional realization if and only if $\lambda$ is a quasi-exponential function, whereas $\varphi_{0}$ is allowed to be any smooth field.

All this was done for the special case of of a scalar Wiener process. For the general case when there are $m$ driving Wiener process, and each volatility is a constant direction volatility, i.e.

$$
\sigma_{i}(q, x)=\varphi_{i}(q) \lambda_{i}(x), \quad 1, \ldots, m,
$$

we are unable to obtain necessary and sufficient conditions (apart, of course, from the abstract one in Theorem 3.1. We can however easily obtain the following sufficient conditions for the existence of a finite dimensional realization.

Proposition 3.4 Under the assumption that $\varphi_{i}(q) \neq 0$ for all $q \in \mathcal{H}$ and $i=1, \ldots, m$, a sufficient condition for the volatility structure (25) to have a finite dimensional realization is that all the functions $\lambda_{i} i=1, \ldots, m$ are quasiexponential functions. The vector fields $\varphi_{1}, \ldots, \varphi_{m}$ are allowed to be any smooth fields.

Proof. In this case the driftr vector field is given by

$$
\mu(q)=\mathbf{F} r-\frac{1}{2} \sum_{i=1}^{m} \varphi_{i}^{2}(q) L_{i}-\frac{1}{2} \sum_{i=1}^{m} \varphi_{i}^{\prime}(q)\left[\lambda_{i}\right] \varphi_{i}(q) \lambda_{i},
$$

where

$$
L_{i}(x)=\lambda_{i}^{2}(x), \quad i=1, \ldots, m .
$$


We thus have to study the Lie algebra generated by the vector fields

$$
\begin{aligned}
\mu(r) & =\mathbf{F} r-\frac{1}{2} \sum_{i=1}^{m} \varphi_{i}^{2}(r) L_{i}-\frac{1}{2} \sum_{i=1}^{m} \varphi_{i}^{\prime}(r)\left[\lambda_{i}\right] \varphi_{i}(r) \lambda_{i} \\
\sigma_{1}(r) & =\varphi_{1}(r) \lambda_{1} \\
& \vdots \\
\sigma_{m}(r) & =\varphi_{m}(r) \lambda_{m} .
\end{aligned}
$$

Under the assumptions of the proposition, we can perform Gaussian elimination to see that the Lie algebra is in fact generated by the much simpler system of vector fields

$$
\begin{aligned}
f_{0}(q) & =\mathbf{F} q-\frac{1}{2} \sum_{i=1}^{m} \Phi_{i}(q) L_{i}, \\
f_{1}(q) & =\lambda_{1} \\
& \vdots \\
f_{m}(q) & =\lambda_{m},
\end{aligned}
$$

where we have used the notation

$$
\Phi_{i}(q)=\varphi_{i}^{2}(q) .
$$

From this it is obvious that the Lie algebra $\{\mu, \sigma\}_{L A}$ is in fact included in the algebra $\left\{\mathbf{F} q, L_{1}, \ldots, L_{m}, \lambda_{1}, \ldots, \lambda_{m}\right\}_{L A}$. For this Lie algebra we have (see the proof of Propostion 3.1) the trivial relation

$$
\begin{aligned}
& \left\{\mathbf{F} q, L_{1}, \ldots, L_{m}, \lambda_{1}, \ldots, \lambda_{m}\right\}_{L A} \\
= & \operatorname{span}\left\{\mathbf{F} q, \mathbf{F}^{n} L_{i}, \mathbf{F}^{n} \lambda_{i} ; i=1, \ldots, m ; n \geq 0\right\} .
\end{aligned}
$$

Finally we may argue as in the proof of Proposition 3.2 to see that

$$
\operatorname{span}\left\{\mathbf{F} q, \mathbf{F}^{n} L_{i}, \mathbf{F}^{n} \lambda_{i} ; i=1, \ldots, m ; n \geq 0\right\}
$$

is finite dimensional if $\lambda_{1}, \ldots, \lambda_{m}$ are quasi exponential.

Remark 3.2 In terms of modelling $F_{0}$ and $\sigma_{0}$ this means that if we consider a volatility structure of the form

$$
\sigma_{0 i}\left(F_{0}, x\right)=\varphi_{0 i}\left(F_{0}\right) \lambda_{i}(x), \quad 1, \ldots, m,
$$

Then, under the nondegeneracy condition $\Phi_{0}^{\prime \prime}\left(F_{0}\right)[\lambda ; \lambda] \neq 0$, we see that a sufficient condition for the existence of an FDR is that $\lambda_{1}, \ldots, \lambda_{M}$ are quasi exponential. 


\section{Invariant manifolds}

Consider a general SDE

$$
\left\{\begin{array}{l}
d q_{t}=\mu\left(q_{t}\right) d t+\sigma\left(q_{t}\right) \circ d W_{t}, \\
q_{0}=q^{0} .
\end{array}\right.
$$

on the space $\mathcal{H}$.

A key concept when dealing with FDRs is the idea of an invariant manifold.

Definition 4.1 A submanifold $\mathcal{G}$ in $\mathcal{H}$ is said to be (locally) invariant under the action of the $S D E$ (27), if for every choice of $q^{0} \in \mathcal{G}$ we have $q_{t} \in \mathcal{G}$ for $0 \leq t \leq \tau\left(q^{0}\right)$, where $\tau$ is a strictly positive stopping time.

To understand this concept, suppose for example that the SDE under study is the futures price equation (15) with an initial point $q^{0}$, and suppose for simplicity that we can (which is often the case) disregard the prefix "local" in the definition. Then, in more pedestrian terms, the invariant manifold $\mathcal{G}$ is a finite dimensional family of (potential) futures price curves, and in fact all futures price curves that will ever be produced by your model will in fact belong to $\mathcal{G}$.

From a more theoretical perspective, the importance of the ivariant manifolds stems from the following central result which was proved in [4].

Theorem 4.1 (Björk and Christensen) Consider the SDE (27). There will exist an FDR for this equation if and only if, for each fixed initial point $q^{0}$, there exists a finite dimensional invariant manifold (typically depending upon the choice of $q^{0}$ ) which contains the point $q^{0}$.

For any given model admitting an FDR, and for a given initial point $q^{0}$, it is of great importance to understand what the invariant manifold looks like.

In [6] a very concrete parameterization of the invariant manifold of an SDE is given, and we need the following definition to describe the parameterization.

Definition 4.2 Let $f$ be a smooth vector field on $\mathcal{H}$, and let $y$ be a fixed point in $\mathcal{H}$. Consider the $O D E$

$$
\left\{\begin{aligned}
\frac{d y_{t}}{d t} & =f\left(y_{t}\right) \\
y_{0} & =y .
\end{aligned}\right.
$$

We denote the solution $y_{t}$ as $y_{t}=e^{f t} y$.

The invariant manifold can now be computed using the following construction from $[6]$. 
Theorem 4.2 (Björk and Svensson) Assume that the Lie algebra $\{\mu, \sigma\}_{L A}$ is spanned by the smooth vector fields $f_{1}, \ldots, f_{d}$. Then, for the initial point $q^{0}$, all curves produced by the model will belong to the induced tangential manifold $\mathcal{G}$, which can be parameterized as $\mathcal{G}=\operatorname{Im}[G]$, where

$$
G\left(z_{1}, \ldots, z_{d}\right)=e^{f_{d} z_{d}} \ldots e^{f_{1} z_{1}} q^{0},
$$

and where the operator $e^{f_{i} z_{i}}$ is given in Definition 4.2

\subsection{An example}

As a simple example of an invariant manifold let us find the invariant manifold of the SDE (15) with deterministic volatility given by

$$
\sigma(x)=\sigma_{0} e^{-c x},
$$

where $c$ and $\sigma_{0}$ are scalar constants There is thus only one driving Wiener process. This volatility is obviously quasi exponential so the condition in Corollary 3.1 is therefore satisfied. Thus there exists an FDR and from Theorem 4.1 we see that there exists an invariant manifold. We now go on to compute the invariant manifold, and to this end we note that if we let $\lambda$ denote the constant vector field defined by $\lambda(x)=e^{-c x}$, we easily obtain

$$
\{\mu, \sigma\}_{L A}=\operatorname{span}\{\mu, \lambda\} .
$$

Next we have to compute the operators $\exp \{\mu t\}$ and $\exp \{\lambda t\}$. Since for this model the Frechet derivative with respect to $q$ of the volatility is zero, i.e. $\sigma_{q}^{\prime}(q, x)=0$, we obtain the following expression for $\mu$ from (19)

$$
\mu(q, x)=\frac{\partial q}{\partial x}(x)-\frac{1}{2} \sigma^{2}(x) .
$$

Define the constant field $h$ by $h(x)=\sigma^{2}(x)$. Then $\exp \{\mu t\}$ is obtained as the solution to

$$
\frac{d q}{d t}=\mathbf{A} q-\frac{1}{2} h
$$

where $\mathbf{A}=\frac{\partial}{\partial x}$. The solution to this linear equation is

$$
q_{t}=e^{\mathbf{A} t} q_{0}-\frac{1}{2} \int_{0}^{t} e^{\mathbf{A}(t-s)} h d s
$$

where the operator $e^{\mathbf{A} t}$ is left translation (see e.g. [3]), i.e. for any continuous real valued function $f$ we have

$$
e^{\mathbf{A} t} f(x)=f(x+t) .
$$

Thus we have that

$$
\left(e^{\mu t} q_{0}\right)(x)=q_{0}(x+t)-\frac{1}{2} H(t, x)
$$


where

$$
\begin{aligned}
H(t, x) & =\int_{0}^{t} e^{\mathbf{A}(t-s)} h(x) d s=\sigma_{0}^{2} \int_{0}^{t} \lambda(x+t-s) d s \\
& =\sigma_{0}^{2} \int_{0}^{t} e^{-2 c(x+t-s)} d s=\frac{\sigma_{0}^{2} e^{-2 c x}}{2 c}\left(1-e^{-2 c t}\right) .
\end{aligned}
$$

Since the vector field $\lambda$ is constant, the corresponding ODE is trivial, and we have

$$
\left(e^{\lambda t} q_{0}\right)=q_{0}+\lambda t .
$$

The parameterization of the invariant manifold generated by the initial curve $q_{0}$ is therefore given as

$$
G\left(z_{0}, z_{1}\right)(x)=q_{0}\left(x+z_{0}\right)-\frac{\sigma_{0}^{2} e^{-2 c x}}{4 c}\left(1-e^{-2 c z_{0}}\right)+e^{-c x} z_{1} .
$$

\section{Construction of finite dimensional realizations}

In this section we describe a method for how to construct a concrete finite dimensional realization of the futures prices, when such a realization exists. As before we will actually be looking at the logarithm of the futures prices, but the results can of course be translated to the futures prices themselves. We basically follow the methodology in [7].

\subsection{The construction algorithm}

Consider a volatility $\sigma: \mathcal{H} \times R_{+} \rightarrow R^{m}$ for which $\{\mu, \sigma\}_{L A}<\infty$, that is consider a volatility such that the futures prices generated by this volatility can generically be realized by means of a finite dimensional SDE. Then a finite dimensional realization can be constructed in the following way:

- Choose a finite number of vector fields $f_{1}, \ldots, f_{d}$ which $\operatorname{span}\{\mu, \sigma\}_{L A}$.

- Compute the invariant manifold $G\left(z_{1}, \ldots, z_{d}\right)$ using Theorem 4.2 .

- We now have that $q=G(Z)$. Make the following Ansatz for the dynamics of the state space variables $Z$

$$
d Z=a(Z) d t+b(Z) \circ d W_{t} .
$$

It must then (see Appendix A) hold that

$$
G_{\star} a=\mu, \quad G_{\star} b=\sigma .
$$

Use the equations in (30) to solve for the vector fields $a$ and $b$. 


\subsection{An example}

As a simple example of how to construct a realization consider again the deterministic volatility given by

$$
\sigma(x)=\sigma_{0} e^{-c x},
$$

where $\sigma_{0}$ and $c$ are scalar constants. In Section 4.1 we showed that the Lie algebra is spanned by $\mu$ and $\lambda$ where $\lambda(x)=\exp (-c x)$, and we also computed invariant manifold. The first two steps in the algorithm given in the previous section have thus been completed. To obtain a finite dimensional realization it remains to find the dynamics of the state space variables. This means solving the equations (30) and since, in this case the Lie algebra is two dimensional, we look for a two dimensional realization of the form

$$
\begin{aligned}
& d z_{0}=a_{0}(z) d t+b_{0}(z) \circ d W_{t} \\
& d z_{1}=a_{1}(z) d t+b_{1}(z) \circ d W_{t},
\end{aligned}
$$

where $z=\left(z_{0}, z_{1}\right)$. We therefore need the Frechet derivative $G^{\prime}$ of $G$. Since $G$ is just a function of the two real variables $z_{0}$ and $z_{1}$, the Frechet derivative $G^{\prime}(z)$ is in this case given by the standard Jacobian

$$
G^{\prime}\left(z_{0}, z_{1}\right)=\left[\frac{\partial G\left(z_{0}, z_{1}\right)}{\partial z_{0}}, \frac{\partial G\left(z_{0}, z_{1}\right)}{\partial z_{1}}\right]
$$

Thus, using the expression (29) for $G$, we see that for any $\left(\alpha_{0}, \alpha_{1}\right) \in R^{2}$ we have

$$
G^{\prime}\left(z_{0}, z_{1}\right)\left(\begin{array}{c}
\alpha_{0} \\
\alpha_{1}
\end{array}\right)(x)=\frac{\partial}{\partial x} q_{0}\left(x+z_{0}\right) \alpha_{0}-\frac{1}{2} h\left(x+z_{0}\right) \alpha_{0}+\lambda_{i}(x) \alpha_{1},
$$

where $h$ is the constant field given by

$$
h(x)=\sigma^{2}(x) .
$$

Recall that for this model $\mu$ is given by

$$
\mu(q)=\mathbf{A} q-\frac{1}{2} h .
$$

If we use that $q=G(z)$ we can obtain an expression for $\mathbf{A} q$, and the equation $G_{\star} a=\mu$ then reads

$$
\begin{aligned}
& \mathbf{A} q_{0}\left(x+z_{0}\right) a_{0}-\frac{1}{2} h\left(x+z_{0}\right) a_{0}+\lambda(x) a_{1} \\
= & \mathbf{A} q_{0}\left(x+z_{0}\right)-\frac{1}{2} h\left(x+z_{0}\right)+\mathbf{A} \lambda(x) z_{1} .
\end{aligned}
$$

Since this equality is to hold for all $x$, and $a$ is not allowed to depend on $x$ it is possible to identify $a$. Using that $\mathbf{A} \lambda=-c \lambda$ we obtain

$$
\begin{aligned}
& a_{0}=1, \\
& a_{1}=-c z_{1},
\end{aligned}
$$


From $G_{\star} b(z)(x)=\sigma(x)$ we obtain the equation

$$
\begin{aligned}
& \mathbf{A} q_{0}\left(x+z_{0}\right) b_{0}-\frac{1}{2} h\left(x+z_{0}\right) b_{0}+\lambda(x) b_{1} \\
= & \sigma_{0} \lambda(x),
\end{aligned}
$$

Therefore we have that

$$
\begin{aligned}
& b_{0}=0, \\
& b_{1}=\sigma_{0} .
\end{aligned}
$$

The dynamics of the state variables are thus given by

$$
\left\{\begin{aligned}
d Z_{0}(t) & =d t \\
d Z_{1}(t) & =-c Z_{1}(t) d t+\sigma_{0} \circ d W_{t} .
\end{aligned}\right.
$$

Since $\sigma_{0}$ is a constant, the Itô-dynamics will look the same, and we have thus proved the following proposition.

Proposition 5.1 Given the initial forward rate curve $q_{0}$ the system generated by the volatility of equation (31) has a finite dimensional realization given by

$$
q_{t}=G\left(Z_{t}\right)
$$

where $G$ is given by

$$
G\left(z_{0}, z_{1}\right)(x)=q_{0}\left(x+z_{0}\right)-\frac{\sigma_{0}^{2} e^{-2 c x}}{4 c}\left(1-e^{-2 c z_{0}}\right)+e^{-c x} z_{1} .
$$

and the dynamics of the state space variables $Z$ are given by

$$
\left\{\begin{array}{l}
d Z_{0}(t)=d t \\
d Z_{1}(t)=-c Z_{1}(t) d t+\sigma d W_{t} .
\end{array}\right.
$$

The interpretation of the state variable $Z_{1}$ in the parameterization in Proposition 5.1 is not clear. There is however considerable freedom in choosing the state variables. Suppose we would like the spot price $\log$ return $R=q(0)$ to be the state variable instead. We have that

$$
R=q(0)=G\left(Z_{0}, Z_{1}\right)(0)=q_{0}\left(Z_{0}\right)-\frac{\sigma_{0}^{2}}{4 c}\left(1-e^{-2 c Z_{0}}\right)+Z_{1},
$$

and thus

$$
Z_{1}=R-q_{0}\left(Z_{0}\right)+\frac{\sigma_{0}^{2}}{4 c}\left(1-e^{-2 c Z_{0}}\right) .
$$

The parameterization of the invariant manifold in terms of $R$ is then given by

$$
\begin{aligned}
q(x) & =\tilde{G}\left(Z_{0}, R\right)(x) \\
& =q_{0}\left(x+Z_{0}\right)-e^{-c x} q_{0}\left(Z_{0}\right)+\frac{\sigma_{0}^{2} e^{-2 c x}}{4 c}\left(1-e^{-2 c Z_{0}}\right)\left(1-e^{-c x}\right)+e^{-c x} R .
\end{aligned}
$$


Using Itô's formula the dynamics of $R$ can be found to be

$$
d R(t)=\left\{\alpha\left[Z_{0}(t)\right]-c R(t)\right\} d t+\sigma_{0} d W_{t},
$$

where

$$
\alpha(z)=q_{0}^{\prime}(z)+c q_{0}(z)-\frac{\sigma_{0}^{2}}{4}\left(1+e^{-2 c z}\right) .
$$

We may now want to change state variabels to the spot price $S$. This is easily done since the parameterization of the invariant manifold with the spot price as state variabel is

$$
q(x)=\bar{G}\left(Z_{0}, S\right)(x)=\tilde{G}\left(Z_{0}, \ln S\right)(x) .
$$

The dynamics of the spot price are given by

$$
d S(t)=\left(\alpha\left[Z_{0}(t)\right]+\frac{\sigma_{0}^{2}}{2}-c \ln S(t)\right) S(t) d t+\sigma_{0} S(t) d W_{t} .
$$

We thus see that we have obtained a well known model for the spot price. It is basically a standard Black-Scholes model with two modifications.

- Instead of having a Wiener process with constant drift as the log return, we now have a mean reverting log reeturn.

- The drift term $\alpha\left[Z_{0}(t)\right]$ allows us to fit the model to the given initial term structure of futures prices.

As far as the log return dynamics are concerned, we thus have a close resemblance to the Hull-White extension of the Vasicek short rate model.

\section{Time varying systems}

So far we have only considered homogeneous systems. In this section we introduce the adjustments needed for the theory to be applicable to time varying systems. Consider the following system of equations for the logarithm of the futures prices

$$
\left\{\begin{aligned}
d q_{t} & =\mu\left(q_{t}, t\right) d t+\sigma\left(q_{t}, t\right) \circ d W_{t}, \\
q_{s} & =q^{0} .
\end{aligned}\right.
$$

The volatility is now of the form $\sigma: \mathcal{H} \times R \times R_{+} \rightarrow R^{m}$. The drift $\mu$ is still given by the expression in (19), except that there is now an explicit time dependence. The definition of a realization for this SDE is given below.

Definition 6.1 We say that the SDE (32) has a (local) d-dimensional realization at $\left(s, q^{0}\right)$, if there exists a point $z_{s} \in R^{d}$, smooth vector fields $a, b_{1}, \ldots, b_{m}$ on some open subset $\mathcal{Z}$ of $R^{d}$, and a smooth (submanifold) map $G: \mathcal{Z} \rightarrow \mathcal{H}$, such that $q$ has the local representation

$$
q_{t}=G\left(Z_{t}\right), \quad t \geq s,
$$


where $Z$ is the solution of the d-dimensional Stratonovich SDE

$$
\left\{\begin{aligned}
d Z_{t} & =a\left(Z_{t}\right) d t+b\left(Z_{t}\right) \circ d W_{t}, \\
Z_{s} & =z_{s}
\end{aligned}\right.
$$

The way to handle the explicit time dependence is to enlarge the state space to include running time as a state variable.

Definition 6.2 Define the following extended objects.

$$
\begin{aligned}
\widehat{\mathcal{H}} & =\mathcal{H} \times R, \\
\hat{q} & =\left[\begin{array}{c}
q \\
t
\end{array}\right], \\
\hat{\mu}(\hat{q}) & =\left[\begin{array}{c}
\mu(q, t) \\
1
\end{array}\right], \\
\hat{\sigma}(\hat{q}) & =\left[\begin{array}{c}
\sigma(q, t) \\
0
\end{array}\right] .
\end{aligned}
$$

We have the following theorem from [6].

Theorem 6.1 (Björk and Svensson) The time varying system (32) has a finite dimensional realization if and only if

$$
\operatorname{dim}\{\hat{\mu}, \hat{\sigma}\}_{L A}<\infty .
$$

\subsection{An example}

In this section we study a real life model for gas futures from the point of view of existence and construction of a finite dimensional realization. The model, introduced in [8] and used on the London market, is defined as

$$
\begin{aligned}
& \frac{d F(t, T)}{F(t, T)}=\alpha_{1} e^{-\beta_{1}(T-t)} d W_{1}(t) \\
& +\left[\alpha_{2} e^{-\beta_{2}(T-t)} \sin (\phi+2 \pi T)+\alpha_{3} e^{-\beta_{3}(T-t)} \sin (\phi+2 \pi t)+\gamma\right] d W_{2}(t) .
\end{aligned}
$$

For ease of exposition, we carry out the calculations for a slightly more general model, after which we apply the results to our particular case. Therefore, consider the model

$$
\frac{d F(t, T)}{F(t, T)}=\sum_{i=1}^{n} \sigma_{i}^{F}(t, T) d W_{i}(t)
$$

or, using the Musiela parameterization

$$
F_{0}(t, x)=F(t, t+x)
$$


the model can be written as

$$
d F_{0}(t, x)=\frac{\partial}{\partial x} F_{0}(t, x) d t+F_{0}(t, x) \sum_{i=1}^{n} \sigma_{i}(t, x) d W_{i}(t),
$$

where $\sigma_{i}(t, x)=\sigma_{i}^{F}(t, t+x)$. As before, we consider log prices, i.e.

$$
q_{t}(x)=\ln F_{0}(t, x) .
$$

The dynamics of this process are

$$
d q_{t}(x)=\left(\frac{\partial}{\partial x} q_{t}(x)-\frac{1}{2} \sum_{i=1}^{n} \sigma_{i}^{2}(t, x)\right) d t+\sum_{i=1}^{n} \sigma_{i}(t, x) d W_{i}(t) .
$$

Since the volatility structure is deterministic the Itô and Stratonovich dynamics will look the same, i.e. we have

$$
d q_{t}(x)=\left(\frac{\partial}{\partial x} q_{t}(x)-\frac{1}{2} \sum_{i=1}^{n} \sigma_{i}^{2}(t, x)\right) d t+\sum_{i=1}^{n} \sigma_{i}(t, x) \circ d W_{i}(t) .
$$

We use the operators

$$
\mathbf{A}=\frac{\partial}{\partial x} \quad \text { and } \quad \mathbf{H}=\frac{\partial}{\partial t}
$$

and the notation

$$
\hat{q}=\left[\begin{array}{l}
q \\
t
\end{array}\right], \quad \hat{\mu}=\left[\begin{array}{c}
\mathbf{A} q-\frac{1}{2} \sum_{i=1}^{n} \sigma_{i}^{2}(t) \\
1
\end{array}\right], \quad \hat{\sigma}_{i}=\left[\begin{array}{c}
\sigma_{i}(t) \\
0
\end{array}\right]
$$

and

$$
\hat{\sigma}(t)=\left[\hat{\sigma}_{1}(t), \ldots, \hat{\sigma}_{n}(t)\right] .
$$

By Theorem 6.1, (34) admits a finite dimensional realization, if and only if

$$
\operatorname{dim}\{\widehat{\mu}, \widehat{\sigma}\}_{L A}<\infty .
$$

To see when this condition holds, we compute the Lie-brackets

$$
\begin{aligned}
{\left[\widehat{\mu}, \widehat{\sigma}_{i}\right] } & =\left[\begin{array}{c}
(\mathbf{A}-\mathbf{H}) \sigma_{i}(t) \\
0
\end{array}\right], \quad i=1, \ldots, n \\
{\left[\widehat{\sigma}_{i}, \widehat{\sigma}_{j}\right] } & =\left[\begin{array}{l}
0 \\
0
\end{array}\right], \quad i, j=1, \ldots n \\
{\left[\widehat{\mu},\left[\widehat{\mu}, \widehat{\sigma}_{i}\right]\right] } & =\left[\begin{array}{c}
(\mathbf{A}-\mathbf{H})^{2} \sigma_{i}(t) \\
0
\end{array}\right], \quad i=1, \ldots n
\end{aligned}
$$

From the above calculations, the following proposition follows. 
Proposition 6.1 The Lie-algebra generated by $\hat{\mu}$ and $\hat{\sigma}$ is given by

$\{\hat{\mu}, \hat{\sigma}\}_{L A}=\operatorname{span}\left\{\left[\begin{array}{c}\frac{\partial}{\partial x} q_{t}(x)-\frac{1}{2} \sum_{i=1}^{n} \sigma_{i}^{2}(t, x) \\ 1\end{array}\right],\left[\begin{array}{c}\left(\frac{\partial}{\partial x}-\frac{\partial}{\partial t}\right)^{k} \sigma_{i}(t, x) \\ 0\end{array}\right] ; i=1, \ldots, n ; k=0,1, \ldots\right\}$.

To see if the model (33) admits a finite dimensional realization, we should therefore calculate

$$
\left(\frac{\partial}{\partial x}-\frac{\partial}{\partial t}\right)^{k} \sigma_{i}(t, x)
$$

for the two components (now written on Musiela form)

$$
\begin{aligned}
& \sigma_{1}(t, x)=\alpha_{1} e^{-\beta_{1} x} \\
& \sigma_{2}(t, x)=\alpha_{2} e^{-\beta_{2} x} \sin (\phi+2 \pi(t+x))+\alpha_{3} e^{-\beta_{3} x} \sin (\phi+2 \pi t)+\gamma,
\end{aligned}
$$

and see if there is a $k<\infty$ (hopefully not too large), such that the vectors

$$
\left\{\left(\frac{\partial}{\partial x}-\frac{\partial}{\partial t}\right)^{k} \sigma_{i}(t, x), i=1,2 ; k=1,2 \ldots\right\}
$$

are linearly dependent. Starting with the first component

$$
\sigma_{1}(t, x)=\alpha_{1} e^{-\beta_{1} x}
$$

and introducing the notation

$$
\mathbf{B}=\left(\frac{\partial}{\partial x}-\frac{\partial}{\partial t}\right)
$$

we see that

$$
\mathbf{B} \alpha_{1} e^{-\beta_{1} x}=-\beta_{1} \alpha_{1} e^{-\beta_{1} x}=-\beta_{1} \sigma_{1}(t, x),
$$

so it is clear that with $k=1$, we already get linearly dependent terms. Continuing with the second volatility factor we get

$$
\begin{aligned}
\mathbf{B}^{0} \sigma_{2}(t, x)= & \alpha_{2} e^{-\beta_{2} x} \sin (\phi+2 \pi(t+x))+\alpha_{3} e^{-\beta_{3} x} \sin (\phi+2 \pi t)+\gamma, \\
\mathbf{B}^{1} \sigma_{2}(t, x)= & -\alpha_{2} \beta_{2} e^{-\beta_{2} x} \sin (\phi+2 \pi(t+x))-\alpha_{3} \beta_{3} e^{-\beta_{3} x} \sin (\phi+2 \pi t) \\
& -2 \pi \alpha_{3} e^{-\beta_{3} x} \cos (\phi+2 \pi t) \\
\mathbf{B}^{2} \sigma_{2}(t, x)= & \alpha_{2} \beta_{2}^{2} e^{-\beta_{2} x} \sin (\phi+2 \pi(t+x))+\left(\beta_{3}^{2}-4 \pi^{2}\right) \alpha_{3} e^{-\beta_{3} x} \sin (\phi+2 \pi t) \\
& +4 \pi \alpha_{3} \beta_{3} e^{-\beta_{3} x} \cos (\phi+2 \pi t) .
\end{aligned}
$$

Letting higher powers of $\mathbf{B}$ operate on $\sigma_{2}$ will only result in linear combinations of the terms represented in the three equations above. We conclude that the four terms needed to generate $\mathbf{B}^{k} \sigma_{2}$ for all $k$ are

$\gamma, \quad e^{-\beta_{2} x} \sin (\phi+2 \pi(t+x)), \quad e^{-\beta_{3} x} \sin (\phi+2 \pi t), \quad$ and $\quad e^{-\beta_{3} x} \cos (\phi+2 \pi t)$. 
By Lemma 3.1 in [6], we can choose the vector fields generating the Lie algebra $\{\hat{\mu}, \hat{\sigma}\}_{L A}$ as

$$
\begin{array}{ll}
\hat{f}_{0}=\left[\begin{array}{cl}
\mathbf{A} q_{t}(x)-\frac{1}{2}\left(\sigma_{1}^{2}(t, x)+\sigma_{2}^{2}(t, x)\right) \\
1
\end{array}\right], & \hat{f}_{3}=\left[\begin{array}{c}
e^{-\beta_{2} x} \sin (\phi+2 \pi(t+x) \\
0
\end{array}\right], \\
\hat{f}_{1}=\left[\begin{array}{c}
\gamma \\
0
\end{array}\right], & \hat{f}_{4}=\left[\begin{array}{c}
e^{-\beta_{3} x} \sin (\phi+2 \pi t) \\
0
\end{array}\right], \\
\hat{f}_{2}=\left[\begin{array}{c}
e^{-\beta_{1} x} \\
0
\end{array}\right], & \hat{f}_{5}=\left[\begin{array}{c}
e^{-\beta_{3} x} \cos (\phi+2 \pi t) \\
0
\end{array}\right] .
\end{array}
$$

To obtain a finite dimensional realization we need to solve a number of differential equations, the first one being

$$
\begin{aligned}
& \frac{d q}{d \tau}=\mathbf{A} q-\frac{1}{2} h \\
& \frac{d t}{d \tau}=1
\end{aligned}
$$

where $h$ is defined by

$$
h(t, x)=\alpha_{1}^{2} e^{-2 \beta_{1} x}+\left[\alpha_{2} e^{-\beta_{2} x} \sin (\phi+2 \pi(t+x))+\alpha_{3} e^{-\beta_{3} x} \sin (\phi+2 \pi t)+\gamma\right]^{2}
$$

and the initial values are $q(0)=q^{*}$ and $t(0)=t^{*}$. If we define $H$ by

$$
H(t, x)=-\frac{1}{2} \int_{0}^{t} h\left(t^{*}+s, x+t-s\right) d s
$$

then the solution to the first differential equation can be written as

$$
\begin{aligned}
q(\tau, x) & =q^{*}(\tau+x)+H(\tau, x), \\
t(\tau) & =t^{*}+\tau .
\end{aligned}
$$

The other differential equations we need to solve are

$$
\frac{d}{d \tau}\left[\begin{array}{l}
q \\
t
\end{array}\right]=\hat{f}_{i}=\left[\begin{array}{c}
f_{i} \\
0
\end{array}\right], \quad \text { for } i=1, \ldots, 5,
$$

with initial values $q(0)=q^{*}$ and $t(0)=t^{*}$. Since the right hand side does not depend on $\hat{q}$, these equations are easy to solve, and the solutions are

$$
\left[\begin{array}{l}
q \\
t
\end{array}\right]=\left[\begin{array}{c}
q^{*}+f_{i} \tau \\
t^{*}
\end{array}\right], \quad i=1, \ldots, 5
$$

Now,

$$
\left[\begin{array}{c}
q_{t} \\
t
\end{array}\right]=\widehat{G}\left(Z_{t}\right)=\left[\begin{array}{l}
G_{0}\left(Z_{t}\right) \\
G_{1}\left(Z_{t}\right)
\end{array}\right]
$$


where the interesting part, $G_{0}$, can be written as

$$
\begin{aligned}
G_{0}\left(z_{i} ; i=0, \ldots, 5\right)(x)= & q^{*}\left(z_{0}+x\right)+H\left(z_{0}, x\right)+\gamma z_{1}+e^{-\beta_{1} x} z_{2} \\
& +e^{-\beta_{2} x} \sin \left(\phi+2 \pi\left(t^{*}+z_{0}+x\right)\right) z_{3} \\
& +e^{-\beta_{3} x} \sin \left(\phi+2 \pi\left(t^{*}+z_{0}\right)\right) z_{4} \\
& +e^{-\beta_{3} x} \cos \left(\phi+2 \pi\left(t^{*}+z_{0}\right)\right) z_{5} .
\end{aligned}
$$

We need the dynamics of $Z$. If

$$
d Z_{i}(t)=a_{i}(z) d t+b_{i}(z) \circ d W_{1}(t)+c_{i}(z) \circ d W_{2}(t), \quad i=0, \ldots, 5
$$

where

$$
Z=\left(\begin{array}{c}
Z_{0} \\
Z_{1} \\
Z_{2} \\
Z_{3} \\
Z_{4} \\
Z_{5}
\end{array}\right)
$$

then by the Itô-Stratonovich formula

$$
d q=\sum_{i=0}^{5} \frac{\partial G}{\partial z_{i}} d Z_{i} .
$$

Thus, we need the partial derivatives of $G_{0}$ with respect to $z_{i}$ for $i=0, \ldots, 5$. These are given by

$$
\begin{aligned}
\frac{\partial G_{0}}{\partial z_{0}}= & \mathbf{A} q^{*}\left(z_{0}+x\right)+\frac{\partial}{\partial z_{0}} H\left(z_{0}, x\right)+2 \pi e^{-\beta_{2} x} \cos \left(\phi+2 \pi\left(t^{*}+z_{0}+x\right)\right) z_{3} \\
& +2 \pi e^{-\beta_{3} x} \cos \left(\phi+2 \pi\left(t^{*}+z_{0}\right)\right) z_{4}-2 \pi e^{-\beta_{3} x} \sin \left(\phi+2 \pi\left(t^{*}+z_{0}\right)\right) z_{5}, \\
\frac{\partial G_{0}}{\partial z_{1}}= & \gamma \\
\frac{\partial G_{0}}{\partial z_{2}}= & e^{-\beta_{1} x} \\
\frac{\partial G_{0}}{\partial z_{3}}= & e^{-\beta_{2} x} \sin \left(\phi+2 \pi\left(t^{*}+x+z_{0}\right)\right), \\
\frac{\partial G_{0}}{\partial z_{4}}= & e^{-\beta_{3} x} \sin \left(\phi+2 \pi\left(t^{*}+z_{0}\right)\right), \\
\frac{\partial G_{0}}{\partial z_{5}}= & e^{-\beta_{3} x} \cos \left(\phi+2 \pi\left(t^{*}+z_{0}\right)\right) .
\end{aligned}
$$


After some reshuffling, the dynamics of $q$ takes the form:

$$
\begin{aligned}
& d q=\left\{\begin{array}{c}
a_{0}\left(\begin{array}{c}
\mathbf{A} q^{*}\left(Z_{0}+x\right)+\frac{\partial}{\partial z_{0}} H\left(Z_{0}, x\right)+2 \pi e^{-\beta_{2} x} \cos \left(\phi+2 \pi\left(t^{*}+Z_{0}+x\right)\right) Z_{3} \\
+2 \pi e^{-\beta_{3} x} \cos \left(\phi+2 \pi\left(t^{*}+Z_{0}\right)\right) Z_{4}-2 \pi e^{-\beta_{3} x} \sin \left(\phi+2 \pi\left(t^{*}+Z_{0}\right)\right) Z_{5}
\end{array}\right) \\
+a_{1} \gamma+a_{2} e^{-\beta_{1} x}+a_{3} e^{-\beta_{2} x} \sin \left(\phi+2 \pi\left(t^{*}+x+Z_{0}\right)\right) \\
+a_{4} e^{-\beta_{3} x} \sin \left(\phi+2 \pi\left(t^{*}+Z_{0}\right)\right)+a_{5} e^{-\beta_{3} x} \cos \left(\phi+2 \pi\left(t^{*}+Z_{0}\right)\right)
\end{array}\right\} d t \\
& +\left\{\begin{array}{c}
b_{0}\left(\begin{array}{c}
\mathbf{A} q^{*}\left(Z_{0}+x\right)+\frac{\partial}{\partial z_{0}} H\left(Z_{0}, x\right)+2 \pi e^{-\beta_{2} x} \cos \left(\phi+2 \pi\left(t^{*}+Z_{0}+x\right)\right) Z_{3} \\
+2 \pi e^{-\beta_{3} x} \cos \left(\phi+2 \pi\left(t^{*}+Z_{0}\right)\right) Z_{4}-2 \pi e^{-\beta_{3} x} \sin \left(\phi+2 \pi\left(t^{*}+Z_{0}\right)\right) Z_{5}
\end{array}\right) \\
+b_{1} \gamma+b_{2} e^{-\beta_{1} x}+b_{3} e^{-\beta_{2} x} \sin \left(\phi+2 \pi\left(t^{*}+x+Z_{0}\right)\right) \\
+b_{4} e^{-\beta_{3} x} \sin \left(\phi+2 \pi\left(t^{*}+Z_{0}\right)\right)+b_{5} e^{-\beta_{3} x} \cos \left(\phi+2 \pi\left(t^{*}+Z_{0}\right)\right)
\end{array}\right\} \circ d W_{1} \\
& +\left\{\begin{array}{c}
c_{0}\left(\begin{array}{c}
\mathbf{A} q^{*}\left(Z_{0}+x\right)+\frac{\partial}{\partial z_{0}} H\left(Z_{0}, x\right)+2 \pi e^{-\beta_{2} x} \cos \left(\phi+2 \pi\left(t^{*}+Z_{0}+x\right)\right) Z_{3} \\
+2 \pi e^{-\beta_{3} x} \cos \left(\phi+2 \pi\left(t^{*}+Z_{0}\right)\right) Z_{4}-2 \pi e^{-\beta_{3} x} \sin \left(\phi+2 \pi\left(t^{*}+Z_{0}\right)\right) Z_{5}
\end{array}\right) \\
+c_{1} \gamma+c_{2} e^{-\beta_{1} x}+c_{3} e^{-\beta_{2} x} \sin \left(\phi+2 \pi\left(t^{*}+x+Z_{0}\right)\right) \\
+c_{4} e^{-\beta_{3} x} \sin \left(\phi+2 \pi\left(t^{*}+Z_{0}\right)\right)+c_{5} e^{-\beta_{3} x} \cos \left(\phi+2 \pi\left(t^{*}+Z_{0}\right)\right)
\end{array}\right\} d W_{2}
\end{aligned}
$$

This must be equal to the dynamics we started out with, namely

$$
\begin{aligned}
& \left\{\begin{array}{c}
\mathbf{A} q^{*}\left(Z_{0}+x\right)+\frac{\partial}{\partial x} H\left(Z_{0}, x\right)-\beta_{1} e^{-\beta_{1} x} Z_{2}-\beta_{2} e^{-\beta_{2} x} \sin \left(\phi+2 \pi\left(t^{*}+x+Z_{0}\right)\right) Z_{3} \\
+2 \pi e^{-\beta_{2} x} \cos \left(\phi+2 \pi\left(t^{*}+x+Z_{0}\right)\right) Z_{3} \\
-\beta_{3} e^{-\beta_{3} x} \sin \left(\phi+2 \pi\left(t^{*}+Z_{0}\right)\right) Z_{4}-\beta_{3} e^{-\beta_{3} x} \cos \left(\phi+2 \pi\left(t^{*}+Z_{0}\right)\right) Z_{5} \\
-\frac{1}{2} h\left(t^{*}+Z_{0}, x\right)
\end{array}\right\} d t \\
& +\alpha_{1} e^{-\beta_{1} x} \circ d W_{1} \\
& +\left(\alpha_{2} e^{-\beta_{2} x} \sin \left(\phi+2 \pi\left(t^{*}+Z_{0}+x\right)+\alpha_{3} e^{-\beta_{3} x} \sin \left(\phi+2 \pi\left(t^{*}+Z_{0}\right)\right)+\gamma\right) \circ d W_{2}\right.
\end{aligned}
$$

We want to find coefficients $a_{i}(z), b_{i}(z), c_{i}(z) ; i=0, \ldots 5$ that solves this equation. We know that there exists at least one solution, and that any solution will 
do. By mere inspection, it is clear that the following is a solution:

$$
a=\left[\begin{array}{c}
1 \\
0 \\
-\beta_{1} z_{2} \\
-\beta_{2} z_{3} \\
2 \pi z_{5}-\beta_{3} z_{4} \\
-\beta_{3} z_{5}-2 \pi z_{4}
\end{array}\right], \quad b=\left[\begin{array}{c}
0 \\
0 \\
\alpha_{1} \\
0 \\
0 \\
0
\end{array}\right], \quad c=\left[\begin{array}{c}
0 \\
1 \\
0 \\
\alpha_{2} \\
\alpha_{3} \\
0
\end{array}\right]
$$

The dynamics of the state space process $Z$ therefore become

$$
\begin{aligned}
& d Z_{0}=d t \\
& d Z_{1}=d W_{2} \\
& d Z_{2}=-\beta_{1} Z_{2} d t+\alpha_{1} \circ d W_{1}, \\
& d Z_{3}=-\beta_{2} Z_{3} d t+\alpha_{2} \circ d W_{2}, \\
& d Z_{4}=\left(2 \pi Z_{5}-\beta_{3} Z_{4}\right) d t+\alpha_{3} \circ d W_{2}, \\
& d Z_{5}=-\left(\beta_{3} Z_{5}+2 \pi Z_{4}\right) d t .
\end{aligned}
$$

Again, the Itô and Stratonovich dynamics will look the same, since there is no randomness in the volatilities.

\section{Spot price realizations}

In factor models of the term structure of futures prices a considerable amount of attention has been given to models where, apart from running time $t$, the spot price $S(t)=F(t, t)=e^{q_{t}(0)}$ is the only state variable. See [5] for references. In the framework of the HJM type futures price models that we are studying, this raises an interesting inverse question, namely what the volatility structure $\sigma(q, x)$ must look like in order to guarantee the existence of a realization with the spot price as the only nontrivial factor or, equivalently, to guarantee that the induced spot price is Markovian. In interest rate theory this problem corresponds to the question about which HJM models can be realized as short rate models, and the problem was solved in [19]. See also [6] and [10]. In the present study we follow the ideas in [6].

As before it is easier to work in terms of $q$ defined in (9) than with the futures prices themselves. The problem is thus to determine when the SDE (15) has a two-dimensional realization in terms of time and $q_{t}(0)$. To simplify the notation we define $R$ as

$$
R(t)=q_{t}(0) .
$$

In order to avoid degenerate cases we need a basic assumption.

Assumption 7.1 We assume that we have only one scalar driving Wiener process, i.e. that $m=1$.

We now have the following fundamental result, which has a parallell in [6]. 
Theorem 7.1 Assume that the model is not deterministic, and consider a given time invariant volatility $\sigma(q, x)$. Then there exists a realization of (15) with time and $q$ as state variables if and only if the Lie bracket vector field $[\mu, \sigma]$ is parallell to the vector field $\sigma$, i.e. if and only if there exists a scalar field $\alpha(q)$ such that the following relation holds (locally) for all $q$.

$$
[\mu, \sigma](q)=\alpha(q) \sigma(q) .
$$

where $\mu$ is the drift of $q$, i.e.

$$
\mu(t, x)=\frac{\partial}{\partial x} q(t, x)-\frac{1}{2} \sigma^{2}(q, x)-\frac{1}{2} \sigma_{q}^{\prime}(q, x)[\sigma(q)] .
$$

If the volatility is time varying of the form $\sigma(q, t, x)$, then (41) is replaced with

$$
\mu_{q}^{\prime}(q, t)[\sigma(q, t)]-\sigma_{q}^{\prime}(q, t)[\mu(q, t)]-\sigma_{t}^{\prime}(q, t) \alpha(q, t) \sigma(q, t) .
$$

Proof. The proof is identical to the corresponding proof in [6]. To give the reader an idea of the technique, we provide the necessity part of the proof. Assume thus that there exists a two dimensional realization where running time is one of the state variables. Then it must have the following form (where $z_{1}$ is time and $z_{2}=q$ )

$$
\begin{aligned}
d z_{1} & =1 \cdot d t+0 \circ d W, \\
d z_{2} & =a(z) d t+b(z) \circ d W, \\
q_{t} & =G\left(z_{t}\right) .
\end{aligned}
$$

In vector notation this reads

$$
d z=A(z) d t+B(z) \circ d W,
$$

where the vector fields $A$ and $B$ are given by

$$
A(z)=\left[\begin{array}{c}
1 \\
a(z)
\end{array}\right], \quad B(z)=\left[\begin{array}{c}
0 \\
b(z)
\end{array}\right] .
$$

The Frechet derivatives (Jacobians) are easily obtained as follows, where subscript denotes partial derivatives.

$$
A^{\prime}(z)=\left[\begin{array}{cc}
0 & 0 \\
a_{1}(z) & a_{2}(z)
\end{array}\right], \quad B^{\prime}(z)=\left[\begin{array}{cc}
0 & 0 \\
b_{1}(z) & b_{2}(z)
\end{array}\right]
$$

Thus the Lie bracket is given by

$$
[A, B]=A^{\prime} B-B^{\prime} A=\left[\begin{array}{c}
0 \\
a_{2} b-b_{2} a-b_{1}
\end{array}\right] .
$$

It is now clear by inspection that we have $[A, B] / / B$, where $/ /$ denotes parallel. On the other hand, because of the relation $q=G(z)$, we also have $\mu=G_{\star} A$ and 
$\sigma=G_{\star} B$. Using the fact that the bracket is preserved under smooth mappings (see Appendix A) we obtain

$$
[\mu, \sigma]=\left[G_{\star} A, G_{\star} B\right]=G_{\star}[A, B] .
$$

Since $[A, B] / / B$ we thus have $G_{\star}[A, B] / / G_{\star} B$, but $G_{\star} B=\sigma$ and we are finished. The sufficiency part is slightly trickier, but can be done. See [6].

Remark 7.1 We will write the condition (41) as

$$
[\mu, \sigma] \| \sigma,
$$

i.e. we will use $\|$ to denote that two fields are parallel.

Remark 7.2 Note that our aim is to have a spot price realization of the futures price model, i.e. a realization in terms of time and $S_{t}$. However if the SDE for $q$ can be realized in terms of time and $R$ we will indeed also have a spot price realization, since the futures prices are given by

$$
F(t, T)=F_{0}(t, T-t)=e^{q(t, T-t)},
$$

and the spot price is related to $R$ in the following way

$$
S(t)=e^{q_{t}(0)}=e^{R_{t}} .
$$

Also note that all results concerning the log futures price volatility $\sigma(q, x)$ can easily be transformed into a corresponding futures price volatility $\sigma_{0}\left(F_{0}, x\right)$ by using the relation in Remark 2.1.

In the following two sections we apply Theorem 7.1 to the special cases studied previously: deterministic and constant direction volatility.

\subsection{Deterministic volatility}

In this section we consider a deterministic, but time varying volatility

$$
\sigma(q, t, x)=\sigma(t, x)
$$

Using (19) we obtain

$$
\mu(q, t, x)=\frac{\partial}{\partial x} q(t, x)-\frac{1}{2} \sigma^{2}(t, x) .
$$

From the above we see that $\mu_{q}^{\prime}=\partial / \partial x$ and $\sigma_{q}^{\prime}=0$. The condition for time varying volatilities in Theorem 7.1 now reads

$$
\sigma_{x}^{\prime}(t, x)-\sigma_{t}^{\prime}(t, x)=\alpha(q, t) \sigma(t, x) .
$$


Therefore we must have that $\alpha(q, t)=\alpha(t)$. Let $g(t, x)=\ln \sigma(t, x)$. The equation can then be written as

$$
g_{x}^{\prime}(t, x)-g_{t}^{\prime}(t, x)=\alpha(t) .
$$

Finally, letting $h(t, x)=g_{x}^{\prime}(t, x)$ and taking derivatives with respect to $x$ we can write

$$
h_{x}^{\prime}(t, x)-h_{t}^{\prime}(t, x)=0 .
$$

The solution to this equation is $h(t, x)=\lambda(t+x)$, where $\lambda$ is an arbitrary function. Going back to $\sigma$ we have

$$
\sigma(t, x)=c(t) e^{\int_{t}^{t+x} \lambda(u) d u},
$$

where $\lambda$ and $c$ are functions, with $c>0$.

Proposition 7.1 Assume that the volatility is deterministic. i.e. $\sigma=\sigma(t, x)$. Then there exists a realization in terms of $R$ if and only if $\sigma$ is of the form

$$
\sigma(t, x)=c(t) e^{\int_{t}^{t+x} \lambda(u) d u},
$$

where $\lambda$ and $c$ are some functions, with $c>0$.

Again we have that $\sigma=\sigma_{0}$ when they are deterministic and the above proposition can be rewritten as

Proposition 7.2 Assume that the futures price volatilities are deterministic. i.e. $\sigma_{0}=\sigma_{0}(t, x)$. Then there exists a spot price realization if and only if $\sigma_{0}$ is of the form

$$
\sigma_{0}(t, x)=c(t) e^{\int_{t}^{t+x} \lambda(u) d u},
$$

where $\lambda$ and $c$ are some functions, with $c>0$.

Remark 7.3 The reader will recognize this result from the corresponding result for short rate realizations in [10] (where completely different rechniques were used).

\subsection{Constant direction volatility}

Now we go on to consider a time invariant constant direction volatility, i.e. a volatility of the form

$$
\sigma(q, x)=\varphi(q) \lambda(x)
$$

For this model we obtain from equation (19)

$$
\mu(q, x)=\frac{\partial}{\partial x} q(x)-\frac{1}{2}\left\{\varphi^{2}(q) \lambda^{2}(x)+\varphi_{q}^{\prime}(q)[\lambda] \varphi(q) \lambda(x)\right\} .
$$


Assume that $\varphi(q) \neq 0$ for all $q \in \mathcal{H}$. Then $[\mu, \sigma] \| \sigma$ if and only if $\left[f_{0}, f_{1}\right] \| \lambda$ where

$$
\left\{\begin{array}{l}
f_{0}(q, x)=\frac{\partial q}{\partial x}(x)-\frac{1}{2} \varphi^{2}(q) \lambda^{2}(x) \\
f_{1}(q, x)=\lambda(x),
\end{array}\right.
$$

We have that

$$
\left[f_{0}, f_{1}\right](q)=\frac{\partial \lambda}{\partial x}-\varphi_{q}^{\prime}(q)[\lambda] \varphi(q) \lambda^{2},
$$

and therefore there exists a realization in terms of $R$ if and only if there exists a scalar field $c(q)$ such that

$$
\frac{\partial \lambda}{\partial x}(x)-\varphi_{q}^{\prime}(q)[\lambda] \varphi(q) \lambda^{2}(x)+c(q) \lambda(x)=0,
$$

for all $q$. Now specialize to the case that $\varphi$ only depends on $q_{t}(0)=R_{t}$, i.e.

$$
\sigma(q, x)=\varphi(R) \lambda(x) .
$$

Equation (44) then becomes

$$
\frac{\partial \lambda}{\partial x}(x)-\varphi_{R}^{\prime}(R) \lambda(0) \varphi(R) \lambda^{2}(x)+\gamma(R) \lambda(x)=0 .
$$

Assume that $\lambda(0)=1$ and set $x=0$ to obtain that

$$
\gamma(R)=\varphi_{R}^{\prime}(R) \varphi(R)-\frac{\partial \lambda}{\partial x}(0) .
$$

Insert this to obtain the equation

$$
\varphi_{R}^{\prime}(R) \varphi(R)=\frac{\lambda^{\prime}(0) \lambda(x)-\lambda^{\prime}(x)}{\lambda(x)-\lambda^{2}(x)} .
$$

Since the left hand side only depends on $R$ and the right hand side only depends on $x$ both sides must equal a constant. If we denote the constant by $a / 2$ we have the following proposition.

Proposition 7.3 Assume that the volatility is of the form $\sigma(q, x)=\varphi(R) \lambda(x)$, and that $\lambda(0)=1$. Then there exists a realization in terms of $R$ if and only if $\varphi^{2}$ is affine in $R$ i.e. of the form

$$
\varphi^{2}(R)=a R+b,
$$

where $a$ and $b$ are constants, and $\lambda$ is the solution to the Riccati equation

$$
\frac{a}{2} \lambda^{2}+\left(\lambda^{\prime}(0)-\frac{a}{2}\right) \lambda-\lambda^{\prime}=0 .
$$


Since for this case we have that

$$
\sigma_{0}(S, x)=\varphi_{0}(S) \lambda(x),
$$

where $\lambda$ is the same constant field as in (45), and

$$
\varphi_{0}(S)=\varphi(\ln S) .
$$

we can rewrite Proposition 7.3 as follows.

Proposition 7.4 Assume that the volatility is of the form $\sigma(q, x)=\varphi(R) \lambda(x)$, and that $\lambda(0)=1$. Then there exists a realization in terms of $R$ if and only if $\varphi^{2}$ is affine in $R$ i.e. of the form

$$
\bar{\varphi}^{2}(S)=a \ln S+b,
$$

where $a$ and $b$ are constants, and $\lambda$ is the solution to the Riccati equation

$$
\frac{a}{2} \lambda^{2}+\left(\lambda^{\prime}(0)-\frac{a}{2}\right) \lambda-\lambda^{\prime}=0 .
$$

\subsection{Generic spot price models}

We will now consider the case when the volatility $\sigma(q)$ only depends on $R$, i.e. when we with a slight abuse of notation can write

$$
\sigma(q, x)=\sigma(R, x),
$$

where $\sigma$ on the right hand side is a smooth function of the two real variables $R$ and $x$.

It turns out that the condition $[\mu, \sigma] \| \sigma$ is rather restrictive for this case, and we have the following result.

Proposition 7.5 Assume that the volatility only depends on $R$ as in (46), then there exists a realization in terms of $R$ (and time) if and only if $\sigma$ factors as

$$
\sigma(R, x)=\varphi(R) \lambda(x) .
$$

Proof. For this model we can compute the drift to be

$$
\mu(q, x)=\frac{\partial}{\partial x} q(x)-\frac{1}{2} \sigma^{2}(R, x)-\frac{1}{2} \sigma_{R}^{\prime}(R, x) g(R),
$$

where $g(R)=\sigma(R, 0)$ and $\sigma_{R}^{\prime}=\partial \sigma / \partial R$. We now compute the Lie bracket $[\mu, \sigma]=\mu_{q}^{\prime}[\sigma(q)]-\sigma_{q}^{\prime}[\mu(q)]$. We have

$$
\begin{aligned}
\mu_{q}^{\prime}[\sigma(q)](x)= & \sigma_{x}^{\prime}(R, x)-\sigma(R, x) \sigma_{R}^{\prime}(R, x) g(R) \\
& -\frac{1}{2}\left\{\sigma_{R}^{\prime \prime}(R, x) g(R)+\sigma_{R}^{\prime}(R, x) g^{\prime}(R)\right\} g(R),
\end{aligned}
$$


and

$$
\sigma_{q}^{\prime}[\mu(q)](x)=\sigma_{R}^{\prime}(R, x) \frac{\partial}{\partial x} q(0)-\frac{1}{2} \sigma_{R}^{\prime}(R, x) g^{2}(R)-\frac{1}{2} \sigma_{R}^{\prime}(R, x) g^{\prime}(R) g(R),
$$

and thus

$$
\begin{aligned}
{[\mu, \sigma](x)=} & \sigma_{x}^{\prime}(R, x)-\sigma(R, x) \sigma_{R}^{\prime}(R, x) g(R) \\
& -\frac{1}{2}\left\{\sigma_{R}^{\prime \prime}(R, x)-\sigma_{R}^{\prime}(R, x)\right\} g^{2}(R)-\sigma_{R}^{\prime}(R, x) \frac{\partial}{\partial x} q(0) .
\end{aligned}
$$

Applying Theorem 7.1 we know that a necessary and sufficient condition for a realization in terms of $R$ (and time) to exist is that $[\mu, \sigma] \| \sigma$. If we let $z=\partial q(0) / \partial x$ we find that there must exist a function $\alpha(z, R)$ such that

$$
\begin{gathered}
\sigma_{x}^{\prime}(R, x)-\sigma(R, x) \sigma_{R}^{\prime}(R, x) g(R) \\
-\frac{1}{2}\left\{\sigma_{R}^{\prime \prime}(R, x)-\sigma_{R}^{\prime}(R, x)\right\} g^{2}(R)-\sigma_{R}^{\prime}(R, x) z=\alpha(z, R) \sigma(R, x)
\end{gathered}
$$

is satisfied for all $z$ and $R$. Take the derivative with respect to $z$ to obtain

$$
-\sigma_{R}^{\prime}(R, x)=\alpha_{z}^{\prime}(z, R) \sigma(R, x) .
$$

From this it follows that $\alpha_{z}^{\prime}(z, R)=f(R)$, and thus we have

$$
\sigma_{R}^{\prime}(R, x)+f(R) \sigma(R, x)=0 .
$$

This is an ODE for $\sigma(R, x)$ with the solution

$$
\sigma(R, x)=\exp \left\{\int_{0}^{R} f(u) d u\right\} \sigma(0, x),
$$

and we see that the volatility factorizes as in (47).

Since we for this case have that $\sigma_{0}(S, x)=\sigma(\ln S, x)$ we can rewrite the proposition in the following way.

Proposition 7.6 Assume that the futures price volatility only depends on $S$, i.e. $\sigma_{0}\left(F_{0}, x\right)=\sigma_{0}(S, x)$, then there exists a spot price realization if and only if $\sigma_{0}$ factors as

$$
\sigma_{0}(S, x)=\varphi(S) \lambda(x) .
$$

\subsection{All generic spot price models are affine}

The following three spot price models are well known from the literature.

$$
\begin{aligned}
d S_{t} & =\alpha(t) S_{t} d t+\sigma S_{t} d W_{t}, \\
d S_{t} & =\left(\alpha(t)-\ln S_{t}\right) S_{t} d t+\sigma S_{t} d W_{t}, \\
d S_{t} & =\left[a_{1}(t)+a_{2}(t) \ln S_{t}\right] S_{t} d t+S_{t} \sqrt{k_{0}+k_{1} \ln S_{t}} d W_{t} .
\end{aligned}
$$


Since all these models (and in fact only these, see [5]) will generate exponentially affine futures prices, the models will be refered as the affine spot price models. In interest rate theory they correspond to the Ho-Lee model, the Hull-White extension of the Vasicek model, and the Hull-White extension of the CIR model respectively. Cecause of the affine structure one can easily (by solving a simple system of ODEs, see [5]) compute the induced futures price volatilities. The structural result is as follows.

Lemma 7.1 The futures price volatilities generated by the affine models (49)(51) all have the structure

$$
\varphi_{0}^{2}(S)=a \ln S+b
$$

where $a$ and $b$ are constants, and $\lambda$ is the solution to the Riccati equation

$$
\frac{a}{2} \lambda^{2}+\left(\lambda^{\prime}(0)-\frac{a}{2}\right) \lambda-\lambda^{\prime}=0
$$

More precisely, the folowing hold.

- For the model (49) it holds that $a=0$ and thus $\lambda(x)=\lambda(0)$ for all $x$, i.e. $\lambda$ is constant.

- For the model (50) it holds that a/2 $=\lambda(0)$ so $\lambda$ is an exponential function.

- For the model (51) it holds that $a / 2 \neq \lambda(0)$, so $\lambda$ solves a Riccati equation.

We recognize the structure above from Proposition 7.4, and we are now ready to state and prove the main theorem of this section.

Theorem 7.2 Assume that the futures price volatilities are of the form

$$
\sigma_{0}\left(F_{0}, x\right)=\sigma_{0}(S, x)
$$

Then the model has a generic spot price realization if and only if it is affine.

Proof. This follows from Proposition 7.4, Proposition 7.6 and Lemma 7.1.

The word "generic" is very important in the statement above (see Remark 2.2 for more details). To understand the geometric picture one can think of the following program.

1. Choose an arbitrary spot price model, say of the form

$$
d S_{t}=a\left(t, S_{t}\right) d t+b\left(S_{t}\right) d W_{t}
$$

with a fixed initial point $S_{0}$.

2. Solve the associated PDE in order to compute futures prices. This will also produce: 
- An initial futures price curve $\hat{q}^{o}(x)$.

- Time invariant futures price volatilities of the form $\sigma_{0}(S, x)$.

3. Now forget about the underlying spot price model, and take the futures price volatility structure $\sigma_{0}(S, x)$ as exogenously given in the futures price equation (7).

4. Initiate the furtures price equation (7) with an arbitrary initial forward rate curve $q^{o}(x)$

The question is now whether the thus constructed futures price model will have a spot price realization. Obviously, if you choose the initial futures price curve $q^{o}$ as $q^{o}=\hat{q}^{o}$, then you are back where you started, and everything is OK. If, however, you choose another initial forward rate curve $q^{o}$, then it is no longer clear that the price will be Markovian (or rather, satisfy a scalar $\mathrm{SDE}$ ). What the theorem above says, is that only the models listed above will be generated by a spot price model for all initial points in a neighborhood of $q^{o}$. If you take another model then a generic choice of the initial futures price curve will produce a futures price process which is not generated by a scalar (time dependent) SDE for the spot price.

\section{A Some Facts from Differential Geometry}

In this Appendix we recall soem basic concepts and results from diferential geometry. For more details see [6].

Consider a real Hilbert space $\mathcal{H}$. By an $n$-dimensional distribution we mean a mapping $F$ which, to every $r$ in an open subset $V$ of $\mathcal{H}$, associates an $n$-dimensional subspace $F(r) \subseteq \mathcal{H}$. A collection $f_{1}, \ldots, f_{n}$ of vector fields on $\mathcal{H}$ generates (or spans) $F$ if it holds that $\operatorname{span}\left\{f_{1}(r), \ldots, f_{n}(r)\right\}=F(r)$ for every $r$, where span denotes the linear hull over the real field. The distribution is smooth if there exists smooth (i.e. $C^{\infty}$ ) vector fields $f_{1}, \ldots, f_{n}$ spanning $F$. If $F$ and $G$ are distributions and $G(r) \subseteq F(r)$ for all $r$ we say that $F$ contains $G$, and we write $G \subseteq F$. The dimension of a distribution $F$ is defined pointwise as $\operatorname{dim} F(r)$.

Let $f$ and $g$ be smooth vector fields on $U$. Their Lie bracket is the vector field

$$
[f, g](r)=f^{\prime}(r) g(r)-g^{\prime}(r) f(r),
$$

where $f^{\prime}(r)$ denotes the Frechet derivative of $f$ at $r$, and correspondingly for $g$. We will sometimes write $f^{\prime}(r)[g(r)]$ instead of $f^{\prime}(r) g(r)$ to emphasize that the Frechet derivative is operating on $g$. A distribution $F$ is called involutive if for all smooth vector fields $f$ and $g$ in $F$, their lie bracket also lies in $F$, i.e.

$$
[f, g](r) \in F(r) \quad \forall r .
$$

We are now ready to define the concept of a Lie algebra which will play a central role in what follows. 
Definition A.1 Let $F$ be a smooth distribution on $\mathcal{H}$. The Lie algebra generated by $F$, denoted by $\{F\}_{L A}$, is defined as the minimal (under inclusion) involutive distribution containing $F$.

When trying to determine a concrete Lie algebra the following observations often come in handy.

Lemma A.1 Consider the vector fields $f_{1}, \ldots, f_{k}$ as given. Then the Lie algebra $\left\{f_{1}, \ldots, f_{k}\right\}_{L A}$ remains unchanged under the following operations.

- The vector field $f_{i}$ may be replaced by $\alpha f_{i}$, where $\alpha$ is any smooth nonzero scalar field.

- The vector field $f_{i}$ may be replaced by

$$
f_{i}+\sum_{j \neq i} \alpha_{j} f_{j},
$$

where $\alpha_{1}, \ldots, \alpha_{k}$ are any smooth scalar fields.

Let $F$ be a distribution on $\mathcal{H}$ and let $\varphi: \mathcal{H} \rightarrow \mathcal{K}$ be a diffeomorphism between the two Hilbert spaces $\mathcal{H}$ and $\mathcal{K}$. Then we can define a new distribution $\varphi_{\star} F$ on $\mathcal{K}$ by

$$
\left(\varphi_{\star} F\right)(\varphi(r))=\varphi^{\prime}(r) F(r) .
$$

Similarly, for any smooth vector field $f \in C^{\infty}(U, X)$, we define the field $\varphi_{\star} f$ by $\varphi_{\star} f=\left(\varphi^{\prime} \circ \varphi^{-1}\right)\left(f \circ \varphi^{-1}\right)$. By a straightforward calculation one verifies easily that

$$
\varphi_{\star}[f, g]=\left[\varphi_{\star} f, \varphi_{\star} g\right]
$$

\section{References}

[1] Amin, K. I., NG, V., And Pirrong, S. Valuing energy derivatives. In Managing Energy Price Risk. Risk Publications, 1995, pp. 57-70.

[2] BJörk, T. Arbitrage Theory in Continuous Time. Oxford University Press, 1998.

[3] BJörk, T. A geometric view of interest rate theory. In Option Pricing, Interest Rates and Risk Management., E. Jouini, J. Cvitanic, and M. Musiela, Eds. Cambridge University Press, 2001.

[4] Björk, T. \& Christensen, B.J. (1997) Interest rate dynamics and consistent forward rate curves. To appear in Mathematical Finance.

[5] BJÖRK, T., And LandÉN, C. On the term structure of and futures and forward prices. In Matematicaql Finance - Bachelier Congress 2000. Springer Verlag, 2001. 
[6] BjöRk, T., And Svensson, L. On the existence of finite dimensional realizations for nonlinear forward rate models. Mathematical Finance 11, 2 (2001), 205-243.

[7] BJÖRK, T., AND LANDÉN, C. On the construction of finite dimensional realizations for nonlinear forward rate models. Finance and Stochastics 6, 3 (2002), 303-331.

[8] BLIX, M. A gas futures volatility model. Work in progress.

[9] Brace, A., And Musiela, M. A multifactor Gauss Markov implementation of Heath, Jarrow, and Morton. Mathematical Finance 4 (1994), 259283.

[10] Carverhill, A. When is the spot rate Markovian? Mathematical Finance 4 (1994), 305-312.

[11] Cortazar, G., And Schwartz, E. The valuation of commodity contingent claims. Journal of Derivatives (1994), 27-39.

[12] Cox, J., Ingersold, J., And Ross, S. A theory of the term structure of interest rates. Econometrica 53 (1985), 385-408.

[13] Duffie, D., And Kan, R. A yield factor model of interest rates. Mathematical Finance 6, 4 (1996), 379-406.

[14] Filipović, D., and Teichmann, J. Existence of finite dimensional realizations for stochastic equations, 2001. Forthcoming in J. Funct. Anal.

[15] Filipović, D., and Teichmann, J. On finite dimensional term structure models, 2002. Working paper.

[16] Gibson, R., and Schwartz, E. Stochastic convenience yield and the pricing of oil contingent claims. Journal of Finance 45, 3 (1990), 959-976.

[17] Hilliard, J., AND Reis, J. Valuation of commodity futures and options under stochastic convenience yields, interest rates, and jump diffusion in the spot. JFQA 33, 1 (1998), 61-86.

[18] Hull, J., And White, A. Pricing interest-rate-derivative securities. Review of Financial Studies 3 (1990), 573-592.

[19] Jeffrey, A. Single factor Heath-Jarrow-Morton term structure models based on Markov spot interest rate dynamics. Journal of Financial and Quantitative Analysis 30 (1995), 619-642.

[20] LandÉn, C. Spot price realizations of futures price term structures. Tech. rep., Department of Mathematics, KTH, Stockholm, 2000.

[21] Miltersen, K., And Schwartz, E. Pricing of options on commodity futures with stochastic term structures of convenience yields and interest rates. JFQA 33 (1998), 33-59. 
[22] Musiela, M. Stochastic PDE:s and term structure models. Preprint, (1993).

[23] Reisman, H. Movements of the term structure of commodity futures and the pricing of commodity claims. Haifa University, Israel, (1991).

[24] Schwartz, E. The stochastic behaviour of commodity prices. Journal of Finance 52, 3 (1997), 923-973. 